Canadian Journal of Physics

Canadian

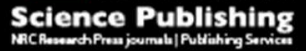

Revue canadienne de physique

\title{
Anisotropic solution in phantom cosmology via Noether symmetry approach
}

\begin{tabular}{|r|l|}
\hline Journal: & Canadian Journal of Physics \\
\hline Manuscript ID & cjp-2017-0765.R2 \\
\hline Danuscript Type: & Article \\
\hline Complete List of Authors: & $\begin{array}{l}\text { Basaran Oz, I.; Akdeniz Universitesi, Department of Physics } \\
\text { Kucukakca, Y.; Akdeniz Universitesi, Department of Physics } \\
\text { Unal, N.; Akdeniz University, Physics Department }\end{array}$ \\
\hline Keyword: & $\begin{array}{l}\text { LRS Bianchi Type I space-time, Phantom cosmology, Noether symmetry } \\
\text { approach, Dark energy, Conserved quantity }\end{array}$ \\
\hline $\begin{array}{r}\text { Is the invited manuscript for } \\
\text { consideration in a Special } \\
\text { Issue? : }\end{array}$ & 33rd International Physics Conference of Turkish Physical Society \\
\hline &
\end{tabular}

SCHOLARONE $^{\text {m }}$

Manuscripts 


\title{
Anisotropic solution in phantom cosmology via Noether symmetry approach
}

\author{
I. Basaran Oz, ${ }^{*}$ Y. Kucukakca ${ }^{\dagger}$ and N. Unal ${ }^{\ddagger}$ \\ Department of Physics, Akdeniz University, Antalya, Turkey
}

(Dated: December 19, 2017)

\begin{abstract}
In this study, we consider a phantom cosmology in which scalar field is minimally coupled to gravity. For anisotropic locally rotational symmetric (LRS) Bianchi type I space-time, we have used the Noether symmetry approach to determine the potential of such a theory. It is shown that the potential must be in the trigonometric form as a function of the scalar field. We solved the field equations of the theory using the result obtained from the Noether symmetry. Our solution shown that the universe has an accelerating expanding phase.

Keywords: LRS Bianchi Type I space-time, Phantom cosmology, Noether symmetry approach, Dark energy, Conserved quantity

PACS numbers: 04.50.+h, 04.40.Dg, 97.60.Gb
\end{abstract}

\section{Introduction}

Since the end of the last century, the analysis of the observational studies, such as supernovae type Ia [1], cosmic microwave background (CMB) [2] etc., show that our universe is expanding with acceleration. The general theory of relativity is insufficient in the issue of explanation of this accelerated expansion phenomenon [3]. This observational data indicate that the present universe is dominated by a fluid with negative pressure, called dark energy (DE) which causes accelerated expansion. The simplest DE candidate is the cosmological constant with the equation of state $(\mathrm{EoS})$ parameter, $w=-1$. But it is not very popular due to its fine tuning and coincidence problem $[4,5]$. As a result of such problems, one of the alternative approach to address the cosmic acceleration issue is to introduce various scalar fields of matter in Einstein gravity, such as quintessence, phantom fields, tachyon field, Chaplygin gas etc. $[4,6]$. Phantom fluid was first investigated in the current cosmological context by Caldwell [7]. Thus, it is possible to construct exact phantom-like cosmological models where all characteristic areas of cosmic evolution are achieved [8].

Observational studies have shown that the cosmic microwave background is the radiation left over from the hot Big Bang. Its temperature is extremely uniform, but has tiny temperature variations or fluctuations (at the part per million level) [9]. Bianchi type space-times are homogeneous but anisotropic space-times [10, 11]. Besides, many authors have considered anisotropic cosmological solutions as possible models for the beginning of the universe which isotropize very rapidly due to particle production in the course of their expansion [12]. Therefore, there are many cosmological studies in the literature, based on Bianchi type space-times [13-18].

Since extended theory of gravities have a nonlinear differential equations, solutions are not very easy. We used the Noether symmetry approach can be used to find solution to the equations of such theories [19,20]. Noether symmetry approach which is directly related to the presence of conserved quantities, can enable us to choose the potential in a dynamical way, and might be able to reduce the number of dynamical variables of the system of differential equations due to possible cyclic variables $[14,16-18,21-24]$. In the present work, we explore the Noether symmetries in phantom cosmology for anisotropic space-time.

This paper is organized as follows. In Sect. 2, we present the field equations in phantom cosmology for the locally rotational symmetric (LRS) Bianchi type I space-time. In Sect. 3, we search the Noether symmetry of the Lagrangian of such a cosmology and give the solution of the field equations by using this approach. Finally, in Sec. 4 , we conclude with a brief summary of the obtained result.

\section{Field equations}

The action that involves gravity minimally coupled with a scalar field is given by [25]

$$
S=\int d^{4} x \sqrt{-g}\left[\frac{R}{2 \kappa}-\frac{1}{2} \phi_{; \mu} \phi^{; \mu}-V(\phi)+\mathcal{L}_{m}\right],
$$

\footnotetext{
*Electronic address: isilbasaran@akdeniz.edu.tr

${ }^{\dagger}$ Electronic address: ykucukakca@akdeniz.edu.tr

${ }^{\ddagger}$ Electronic address: nuriunal@akdeniz.edu.tr
} 
where $\kappa=8 \pi G / c^{4}, \mathcal{L}_{m}$ is the standard matter Lagrangian, $V(\phi)$ is the potential function of the scalar field. We consider the line element as LRS Bianchi type I space-time in the form [13]

$$
d s^{2}=d t^{2}-A(t)^{2} d x^{2}-B(t)^{2}\left(d y^{2}+d z^{2}\right) .
$$

Here, $A(t)$ and $B(t)$ are the metric potentials which are functions of cosmic time. From (2), we see that the metric potentials are different in different directions. Using the metric (2), from the action (1), we find the following point-like Lagrangian,

$$
\mathcal{L}=-2 A \dot{B}^{2}-4 B \dot{A} \dot{B}-A B^{2}\left(\frac{1}{2} \dot{\phi}^{2}+V(\phi)\right),
$$

Here, a dot indicates differentials with respect to cosmic time $t$. We have taken $\mathcal{L}_{m}=0$, ignored the contribution of the standard matter and considered the scalar field. For a spatially homogeneous space-time, the proportion of shear scalar and expansion scalar is constant. The natural harmony with homogeneous expansion leads to a constant ratio $[15,26]$. Thus, we can use a power-law relation $A=B^{m} m \neq 0,1$, where $m$ is a constant anisotropic parameter which represents the deviation of the anisotropic universe model from isotropic. The case $m=1$, refers to the isotropic model of the universe, which has previously been discussed in the literature [27].

Consequently, using this condition, Lagrangian (3) reduces to form as

$$
\mathcal{L}=-(2 m+1) B^{m} \dot{B}^{2}-B^{m+2}\left(\frac{1}{2} \dot{\phi}^{2}+V(\phi)\right) .
$$

It is well known that the Euler-Lagrange equations for a dynamical system are

$$
\frac{d}{d \tau}\left(\frac{\partial \mathcal{L}}{\partial \dot{q}^{i}}\right)-\frac{\partial \mathcal{L}}{\partial q^{i}}=0
$$

where $q^{i}$ are the generalized coordinates of the configuration space with $\dot{q}^{i}$ are the generalized velocities. The configuration space of the Lagrangian (4) is $T(B, \phi)$ with tangent space $T Q(B, \phi, \dot{B}, \dot{\phi})$. To get the field equations, we use the Euler-Lagrange equations (5) for the metric coefficient $B$ and scalar field $\phi$. Thus we have

$$
\begin{array}{r}
(2 m+1)\left[m \frac{\dot{B}^{2}}{B^{2}}+2 \frac{\ddot{B}}{B}\right]-(m+2)\left(\frac{1}{2} \dot{\phi}^{2}+V(\phi)\right)=0, \\
(m+2) \frac{\dot{B}}{B} \frac{\dot{\phi}}{\phi}+\frac{\ddot{\phi}}{\phi}-\frac{V^{\prime}(\phi)}{\phi}=0, \quad(2 m+1) \frac{\dot{B}^{2}}{B^{2}}+\frac{1}{2} \dot{\phi}^{2}-V(\phi)=0,
\end{array}
$$

where a prime denotes derivatives with respect to scalar field $\phi$. The last equation between them is the independent equation obtained by the fact that the energy function $E_{\mathcal{L}}=\dot{q}^{i} \frac{\partial \mathcal{L}}{\partial \dot{q}^{i}}-\mathcal{L}$ is zero. In order to solve these equations we need to determine the form of the potential. To do this, we utilize the Noether symmetry approach in the following section.

\section{Noether symmetry and exact solution}

The Noether symmetry approach is a useful method to obtain the conserved quantities of the dynamical systems. This approach has been studied for many extended theories in cosmology[14, 19, 21, 28-35]. The Noether theorem states that if the Lie derivative of a given Lagrangian $\mathcal{L}$, dragging along a vector field $\mathrm{X}$ vanishes

$$
£_{\mathbf{X}} \mathcal{L}=0,
$$

where $£_{\mathbf{X}}$ stands for Lie derivative with respect to $\mathcal{L}$. Then, $\mathbf{X}$ is said to be a symmetry for the dynamics derived from the Lagrangian and it generates a conserved current [36]. The vector field for the Lagrangian (4) is given by

$$
\mathbf{X}=\alpha \frac{\partial}{\partial B}+\beta \frac{\partial}{\partial \phi}+\dot{\alpha} \frac{\partial}{\partial \dot{B}}+\dot{\beta} \frac{\partial}{\partial \dot{\phi}},
$$

where $\alpha$ and $\beta$ depend on $B$ and $\phi$. From the Lagrangian (4), the Noether symmetry condition (6) yields the following set of system of equations

$$
\begin{aligned}
m \alpha+2 B \frac{\partial \alpha}{\partial B}=0, & (m+2) \alpha+2 B \frac{\partial \beta}{\partial \phi}=0, \\
2(2 m+1) \frac{\partial \alpha}{\partial \phi}+B^{2} \frac{\partial \beta}{\partial B}=0, & (m+2) \alpha V+B \beta V^{\prime}=0 .
\end{aligned}
$$


After some calculations, the solutions of the equations (8) are found as follows

$$
\alpha=c_{1} B^{-\frac{m}{2}} \operatorname{cosk} \phi, \quad \beta=-c_{1} \sqrt{4 m+2} B^{-\frac{(m+2)}{2}} \sin k \phi, \quad V=\frac{c_{1}^{2}}{2}[1-\cos 2 k \phi]
$$

where we define $k=\frac{m+2}{2 \sqrt{4 m+2}}$ and $c_{1}$ is an integration constant. Following the procedure given by [36], we can introduce the new variables as

$$
u=b_{0} B^{\frac{(m+2)}{2}} \operatorname{sink} \phi, \quad z=\frac{2}{c_{1}(m+2)} B^{\frac{(m+2)}{2}} \operatorname{cosk} \phi
$$

where $b_{0}$ is an integration constant. In terms of these new variables the Lagrangian (4) becomes,

$$
\mathcal{L}=-\frac{4(2 m+1)}{b_{0}^{2}(m+2)^{2}} \dot{u}^{2}-\frac{c_{1}}{b_{0}^{2}} u^{2}-c_{1}^{2}(2 m+1) \dot{z}^{2} .
$$

Since the new Lagrangian (10) not depend on the $z$ coordinate, $z$ is a cyclic variable. The field equations are obtained from the equation (10) as

$$
\begin{array}{r}
2 c_{1}^{2}(2 m+1) \dot{z}=I_{0}, \quad \frac{4(2 m+1)}{(m+2)^{2}} \ddot{u}-c_{1} u=0 \\
\frac{4(2 m+1)}{b_{0}^{2}(m+2)^{2}} \dot{u}^{2}-\frac{c_{1}}{b_{0}^{2}} u^{2}+c_{1}^{2}(2 m+1) \dot{z}^{2}=0
\end{array}
$$

where $I_{0}$ is a constant of motion. These equations give us the solution as

$$
u(t)=d_{1} e^{\sqrt{2 c_{1}} k t}+d_{2} e^{-\sqrt{2 c_{1}} k t}, \quad z(t)=\frac{I_{0}}{2 c_{1}^{2}(2 m+1)} t+k_{0},
$$

where $d_{1}, d_{2}$ and $k_{0}$ are the integral constants. Inserting the solutions (12) into the last equation of (11), we obtain a constraint equation as $16(2 m+1) c_{1}^{3} d_{1} d_{2}=I_{0}^{2} b_{0}^{2}$. Then the solutions are obtained for the metric potential and the scalar field.

$$
\begin{aligned}
& B(t)=\left[\frac{c_{1}(m+2)^{2}}{4} z(t)^{2}+\left(\frac{u(t)}{b_{0}}\right)^{2}\right]^{1 /(m+2)}, \\
& \left.\phi(t)=\frac{2 \sqrt{4 m+2}}{(m+2)} \operatorname{arccot}\left[\frac{c_{1}(m+2) b_{0}}{2 u(t)}\right) z(t)\right] .
\end{aligned}
$$

Using the relation $A=B^{m}$, the metric potential $A(t)$ is obtained as

$$
A(t)=\left[\frac{c_{1}(m+2)^{2}}{4} z(t)^{2}+\left(\frac{u(t)}{b_{0}}\right)^{2}\right]^{m /(m+2)} .
$$

Now we give some cosmological parameters such as the Hubble parameter, deceleration parameter and equation of state parameter. We find the average scale factor of the universe defined by $a=\left(A B^{2}\right)^{1 / 3}$ as follows

$$
a(t)=\left[\frac{c_{1}(m+2)^{2}}{4} z(t)^{2}+\left(\frac{u(t)}{b_{0}}\right)^{2}\right]^{1 / 3}
$$

The Hubble parameter is found from the well known definition $H=\dot{a} / a$ as follows

$$
H(t)=\frac{1}{3} \frac{\frac{c_{1}}{2}(m+2)^{2} z(t) \dot{z(t)} 2+\frac{2}{b_{0}^{2}} u(t) \dot{u(t)}}{\frac{c_{1}}{4}(m+2)^{2} z(t)^{2}+\frac{u(t)^{2}}{b_{0}^{2}}} .
$$

The deceleration parameter is defined by $q=-a \ddot{a} / \dot{a}^{2}$. It gives us the rate of expansion of the universe that $q>0$ deceleration, $q<0$ acceleration and $q=0$ constant rate of expansion. It is noted that there are many studies related 
to the time varying deceleration parameter for Bianchi type space-time in different gravitational theories [37-39]. In our case the deceleration parameter obtained as

$$
\begin{array}{r}
q=s^{2}\left(-4 c_{1} k^{2} s^{2}\left(d_{2}^{4}+d_{1}^{4} e^{8 \sqrt{2 c_{1}} k t}\right)-2 d_{1} d_{2} e^{4 \sqrt{2 c_{1}} k t}\left(3 n^{2}+(6 \sqrt{2}-1) c_{1} d_{1} d_{2} k^{2} s^{2}\right)\right. \\
-d_{1}^{2} e^{6 \sqrt{2 c_{1}} k t}\left(24 \sqrt{2} c_{1} d_{1} d_{2} k^{2} s^{2}+n^{2}\left(3+2 \sqrt{2 c_{1}} k t\left(2 \sqrt{c_{1}} k^{2} t^{2}-3\right)\right)\right) \\
\left.-d_{2}^{2} e^{2 \sqrt{2 c_{1}} k t}\left(24 \sqrt{2} c_{1} d_{1} d_{2} k^{2} s^{2}+n^{2}\left(3+2 \sqrt{2 c_{1}} k t\left(2 \sqrt{c_{1}} k^{2} t^{2}+3\right)\right)\right)\right) \\
/\left(-2 \sqrt{c_{1}}\left(d_{2}^{2}-d_{1}^{2} e^{4 \sqrt{2 c_{1}} k t}\right) k s^{2}+e^{2 \sqrt{2 c_{1}} k t} n^{2} t\right)^{2}
\end{array}
$$

where we define $n=b_{0} I_{0}(m+2)$ and $s=4 c_{1}(2 m+1)$. The equation of state parameter is defined by $w=\rho / p$, where $\rho=1 / 2 \dot{\phi}^{2}-V(\phi)$ and $p=1 / 2 \dot{\phi}^{2}+V(\phi) . \omega=0$ case of the state equation parameter corresponds to the matter dominant universe, $\omega=1 / 3$ case radiation dominant universe and $\omega=-1$ case vacuum energy dominant universe. Besides, for $w<-1$ the phantom phase is observed, and for $-1<w<-1 / 3$ the phase is described by quintessence For our case the equation of state parameter obtained as

$$
\begin{array}{r}
w=-\left(12 c_{1} k^{2} s^{4}\left(d_{2}^{4}+d_{1} 4 e^{8 \sqrt{2 c_{1}} k t}\right)+e^{4 \sqrt{2 c_{1}} k t}\left(12 d_{1} d_{2} s^{2}\left(n^{2}+24(4 \sqrt{2}-1) c_{1} d_{1} d_{2} k^{2} s^{2}\right)+n^{4} t^{2}\right)\right. \\
+2 d_{1}^{2} e^{6 \sqrt{2 c_{1}} k t} s^{2}\left(3 n^{2}+(2-6 \sqrt{2}) \sqrt{c_{1}} n^{2} k t+4 \sqrt{2} c_{1} k^{2}\left(6 d_{1} d_{2} s^{2}+n^{2} t\right)\right) \\
\left.+2 d_{2}^{2} e^{2 \sqrt{c_{1}} k t} s^{2}\left(3 n^{2}+(6 \sqrt{2}-2) \sqrt{c_{1}} n^{2} k t+4 \sqrt{2} c_{1} k^{2}\left(6 d_{1} d_{2} s^{2}+n^{2} t\right)\right)\right) \\
/\left(3\left(-2 \sqrt{c_{1}}\left(d_{2}^{2}-d_{1}^{2} e^{4 \sqrt{2 c_{1}} k t}\right) k s^{2}+e^{2 \sqrt{2 c_{1}} k t} n^{2} t\right)^{2}\right) .
\end{array}
$$

We plotted the graphics to analyze the evolution of the cosmological parameter with respect to cosmic time. Fig. (1) represents increasing behavior of the average scale factor indicating expansion of the universe. From Fig. (2), we observed that the deceleration parameter remains always negative and decrease as a function of time. The negative value of the deceleration parameter indicates the present acceleration of the universe. Fig. (3) shows that the values of the equation of state parameters of scalar field are smaller than -1 . Therefore, we have a phantom dark energy model.

\section{Conclusion}

In the present work, a phantom cosmology in which scalar field is minimally coupled to gravity model has been studied using the Noether symmetry approach. In the context of LRS Bianchi type I space-time, Noether symmetry is imposed on the Lagrangian (4) of the system and the symmetry vector is obtained. As a result, the potential takes the trigonometric form given by Eq.(9). Then choosing a transformation $\{B, \phi\} \longrightarrow\{u, z\}$, so that one of the transformed variables $z$ becomes cyclic for the Lagrangian, we obtained some solution for the new field equations. We give the graphical behavior of the solutions in Figs. (1)-(3), which include some cosmological parameter such as scale factor, the deceleration parameter and the equation of state parameter. From Fig. (3) we have the phantom phase of the universe. Furthermore, while $t \rightarrow \infty, w \rightarrow-1$, so that the universe dominated by the vacuum energy. 



FIG. 1: Plots of the average scale factor $a$ FIG. 2: Plots of the deceleration paramewith respect to cosmic time $t$. ter $q$ with respect to cosmic time $t$.



FIG. 3: Plots of the equation of state parameter $w$ with respect to cosmic time $t$.

We take $I_{0}=1, k_{0}=0 ; d_{1}=0.02 ; d_{2}=1 ; c_{1}:=1 ; b_{0}=1 ; m=1.00672$.

[1] A. G. Riess et al. (Supernova Search Team), Astrophys. J. 116, 1009 (1998).

[2] D. N. Spergel et al. (WMAP), ApJS 148, 175 (2003).

[3] S. Weinberg, Gravitation and cosmology: principles and applications of the general theory of relativity (Wiley, 1972), ISBN 9780471925675.

[4] E. J. Copeland, M. Sami, and S. Tsujikawa, Int. J. Mod.Phys. D 15, 1753 (2006).

[5] R. Durrer and R. Maartens, Gen. Relativ. Gravit. 40, 301 (2008).

[6] K. Bamba, S. Capozziello, S. Nojiri, and S. D. Odintsov, Astrophys. Space Sci. 342, 155 (2012).

[7] R. Caldwell, M. Kamionkowski, and N. Weinberg, Phys. Rev. Lett. 91, 071301 (2003).

[8] S. Capozziello, S. Nojiri, and S. D. Odintsov, Phys. Lett. B B632, 597 (2006).

[9] G. Hinshaw et al., ApJS 208, 19 (2013).

[10] G. F. R. Ellis, Gen. Relativ. Gravit. 38, 1797 (2006).

[11] J. D. Barrow and R. Maartens, Phys. Rev. D 59, 043502 (1998).

[12] Y. B. Zel'Dovich and A. A. Starobinskii, Sov. J. Exp. Theor. Phys. 34, 1159 (1972).

[13] A. Pradhan and A. Kumar, Int. J. Mod. Phys. D 10, 291 (2001).

[14] Y. Kucukakca, U. Camci, and I. Semiz, Gen. Relativ. Gravit. 44, 1893 (2012).

[15] W. Xing-Xiang, Chin. Phys. Lett. 21, 1205-1207 (2004).

[16] U. Camci and Y. Kucukakca, Phys. Rev. D 76, 084023 (2007).

[17] U. Camci, A. Yildirim, and I. B. Oz, Astropart. Phys. 76, 29 (2016).

[18] M. F. Shamir and F. Kanwal, Eur. Phys. J. C 77, 286 (2017).

[19] R. de Ritis, G. Marmo, G. Platania, C. Rubano, P. Scudellaro, and C. Stornaiolo, Phys. Rev. D 42, 1091 (1990).

[20] S. Capozziello and R. de Ritis, Phys. Lett. A 177, 1 (1993).

[21] Y. Kucukakca and U. Camci, Astrophys. Space Sci. 338, 211 (2012).

[22] S. Capozziello, G. Marmo, C. Rubano, and P. Scudellaro, Int. J. Mod. Phys. D 06, 491 (1997).

[23] S. Basilakos, M. Tsamparlis, and A. Paliathanasis, Phys. Rev. D 83, 103512 (2011).

[24] A. Paliathanasis, S. Basilakos, E. N. Saridakis, S. Capozziello, K. Atazadeh, F. Darabi, and M. Tsamparlis, Phys. Rev. D 89, $104042(2014)$.

[25] P. Singh, M. Sami, and N. Dadhich, Phys. Rev. D 68, 023522 (2003).

[26] C. B. Collins, E. N. Glass, and D. A. Wilkinson, Gen. Relativ. Gravit. 12, 805 (1980).

[27] S. Capozziello, E. Piedipalumbo, C. Rubano, and P. Scudellaro, Phys. Rev. D D80, 104030 (2009).

[28] Y. Kucukakca, Eur. Phys. J. C 74, 3086 (2014).

[29] Y. Kucukakca, Eur. Phys. J. C 73, 2327 (2013). 
[30] Y. Kucukakca, Astrophys. Space Sci. 361, 80 (2016).

[31] G. Gecim, Y. Kucukakca, and Y. Sucu, Adv. High Energy Phys. 2015, 567395 (2015).

[32] G. Gecim and Y. Sucu, Adv. High Energy Phys. 2017, 2056131 (2017).

[33] M. Sharif and S. Waheed, J. Cosmol. Astropart. Phys. p. 043 (2013).

[34] M. Sharif and I. Shafique, Phys. Rev. D 90, 084033 (2014).

[35] M. Sharif and H. I. Fatima, J. Exp. Theor. Phys. 122, 104 (2016).

[36] S. Capozziello, R. de Ritis, C. Rubano, and P. Scudellaro, La Rivi. del Nuovo. Cim. 19, 1 (1996).

[37] I. Yılmaz, H. Baysal, and C. Aktaş, Gen. Relativ. Gravit. 44, 2313 (2012).

[38] M. S. Borkar and P. V. Gayakwad, Int. J. Mod. Phys. D 26, 1750061 (2017).

[39] P. K. Sahoo, P. Sahoo, B. K. Bishi, and S. Aygün, New Astron. 60, 80 (2018). 